\title{
Identification of tool wear states with fuzzy classification
}

\author{
LI XIAOLI, GUAN XINPING and WANG HONGRUI
}

\begin{abstract}
A new on-line tool wear states detecting method, with spindle and feed current signal in boring, is presented. By analyzing the effects of tool wear, as well as the cutting parameters on the current signals, the models of the relationship between the current signals and the cutting parameters are established under different tool wear states with partial experimental design and regression analysis. Fuzzy classification method is then used to obtain the membership degree of each tool wear classification with measured spindle and feed current values. Finally, the membership results of the spindle current and feed current are fused by the fuzzy inference method, and the tool wear state may be detected effectively. The validity and reliability of the method are verified by experimental results. The method can be effectively employed in practice.
\end{abstract}

\section{Introduction}

On-line tool wear states detection is a very important technique in CIM; it is essential when realizing a fully automated manufacturing system and preventing tool and workpiece damage. Many researchers have looked for ways to detect tool wear states; a large variety of sensors can be used for tool condition sensing ( $\mathrm{Li}$ and Mathew 1990, Daneshmand and Pak 1983), but only a few are reliable and effective. Indirect methods that rely on the relationship between tool states and measured signals (such as force, acoustic emission, vibration, current, etc.) to estimate the tool wear states have been extensively studied. Among the methods used for detecting the tool condition, motor current sensing is a major one. Mannan et al. (1989) described the feasibility of motor power and current sensing for adaptive control and tool condition monitoring. Mannan and Nilsson (1997) measured the spindle and feed

Authors: Li Xiaoli, P.O. Box 426, Harbin Institute of Technology, Harbin 150001, China; and Guan Xinping and Wang Hongrui, Yan Shan University, PR China. current to estimate the static torque and thrust, in order to monitor the tool condition. The major advantage of using the measurement of motor current to detect a malfunction in the cutting process is that the measuring apparatus does not disturb the machining process. Moreover it can be applied in the manufacturing environment at almost no extra cost (Byrne et al. 1995).

In the paper, measured spindle and feed currents are used to estimate the bore wear states. It is known that current signals depend on the cutting variables (cutting speed $v$, feed speed $f$, the depth of cut $d$ ), and on the tool wear $w$. Moreover, tool wear itself also depends on the cutting variables, so the measured currents are affected by the tool wear directly and by the cutting variables indirectly. This paper presents a new method to estimate bore wear states by current measurement; the models with regression technology are presented over a wide range of cutting condition, and the fuzzy method is used to classify the tool wear states. The key idea in the method is to model the relation ship between the measured current signal value and the bore wear states under different cutting conditions. Based on the above method, tool wear states can be estimated by knowing the cutting parameters and the current signal value. Finally, the fuzzy inference method is presented to fuse the classification result of spindle and feed current signals. Therefore, bore wear states can be estimated by the measured current signal and the known cutting variables. Experimental results show that the method can be effectively employed in practice.

\section{Experimental set-up}

The effects of the cutting variables on the current signal should be studied first, so several experiments 
over a range of cutting conditions were conducted. Figure 1 shows a schematic diagram of the experimental set-up. Cutting tests were performed on a Machine Center Makino-FNC74-A20. The four axes (spindle, $\mathrm{X}, \mathrm{Y}$ and $\mathrm{Z}$ ) of the machine have recalculating ball screw drives and are directly driven by permanent magnet synchronous AC servomotors. The AC servo motor current signals of the Machine Center were measured by a Hall current sensor. The signals were first passed though low-pass filters (cut-off frequency $500 \mathrm{~Hz}$ ), and we sent them to a personal computer via an A/ D converter. Table 1 shows the experimental conditions.

During the experiments, both spindle and feed current amplitude change as tool wear, spindle speed, feed speed and the depth of cut change; the following conclusions are drawn.

It is found that both spindle and feed current increase as tool wear increases; this is due to the increase of friction between tool and workpiece. Moreover, current increase s almost line arly with tool wear. It is found that tool wear has a more significant effect on feed current than spindle current.

It is found that both spindle and feed current increase as the depth of cut increases. Moreover, feed current increases almost linearly as the depth of cut increases, while the spindle current increase is proportional to the square of the depth of cut.

It is found that the current signal increase soverall as the spindle speed increases, but the current fluctuates in the spindle speed range $20-30 \mathrm{~m} / \mathrm{min}$; see figure 2 . The cause of the change in current signal is complex. The main influence is temperature; the effect of temperature is small at low speed, and it increases as spindle speed increases.
It is found that the current signal increases as the feed speed increases; the current also fluctuates (see figure 3). The cause of the change in current signal is complex (Shaw 1984).

In brief, the current signals affects the tool wear, spindle speed, feed speed and depth of cut. When establishing the model of the current signal, the above parameters should be included. It is verified that the spindle current and feed current can be selected as the features relevant to bore wear states.

Table 1. Experimental condition.

Tool Tool material: high speed steel

cutting spindle speed: $15-35(\mathrm{~m} / \mathrm{min})$

conditions

feed speed: $10-30(\mathrm{~mm} / \mathrm{min})$

the depth of cut: $0.35,0.45,0.55,0.8,0.9,1.15 \mathrm{~mm}$ without coolant

Workpiece $45 \#$ quench steel

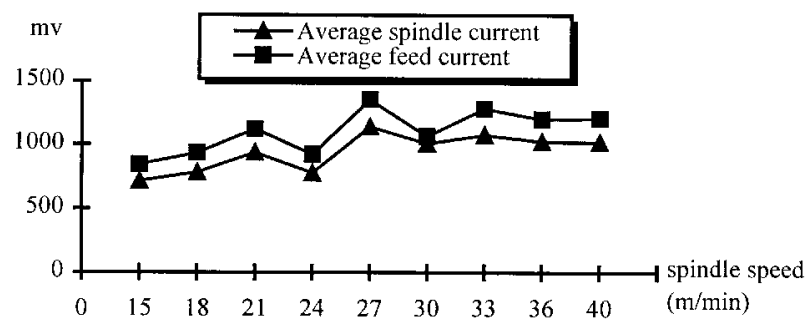

Figure 2. The effect of spindle speed on current signal.

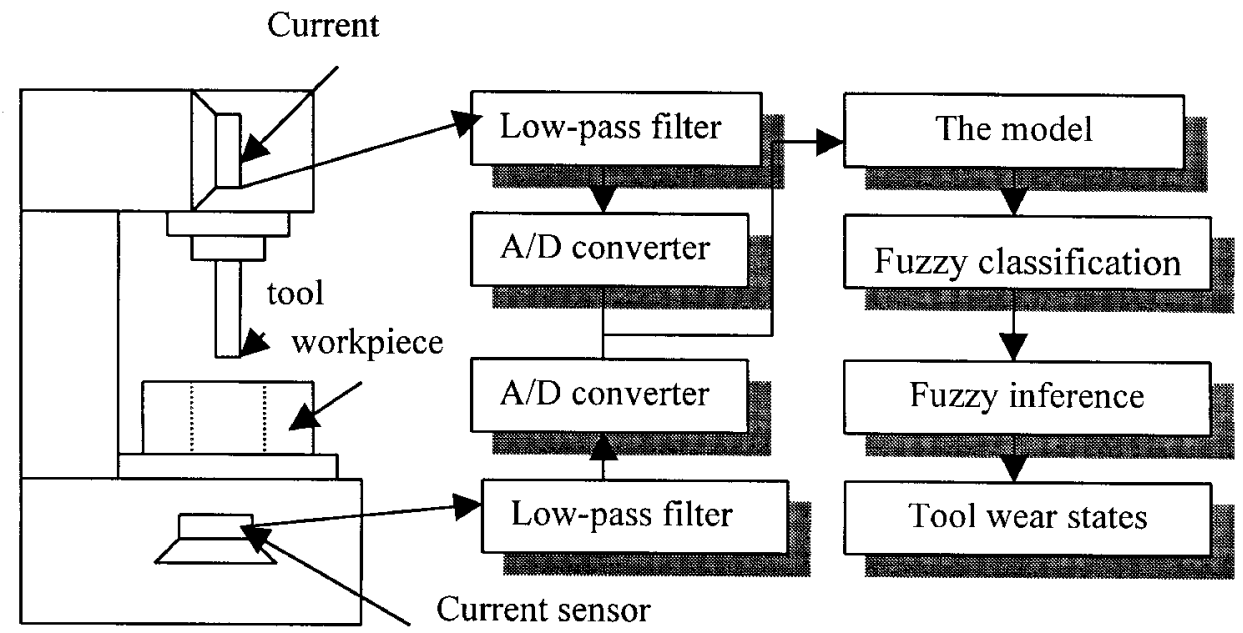

Figure 1. The experimental set-up. 


\section{The model}

Based on the above studies, it is suggested that the effects of tool wear, spindle speed, feed speed and the depth of cut should be taken into account when molding current signals. The bore wear state is divided into A, B, C, D, E and F classification s based on practical requirements; see table 2. Spindle and feed currents are modelled as functions of spindle speed $v(\mathrm{~m} / \mathrm{min})$, feed speed $f(\mathrm{~mm} / \mathrm{min})$ and the depth of cut $d(\mathrm{~mm})$ under different tool wear classifications.

The effect of the cutting variables $v, f$ and $d$ on the spindle and feed current signals under each tool wear classification can be expressed as follows:

$$
\begin{gathered}
I_{S}=K_{s} v^{a_{1}} f^{a_{2}} d^{a_{3}} \\
I_{F}=K_{F} v^{b_{1}} f^{b_{2}} d^{b_{3}}
\end{gathered}
$$

where $I_{S}$ and $I_{F}$ are the spindle and feed current amplitudes, respectively; $K_{S}$ and $K_{F}$ are constant with the tool geometry and workpiece material; the parameters $a_{i}$ and $b_{i}(i=1,2,3)$ are the exponents of the cutting variables. Equation (1) is taken by logarithm, the results under the A, B, C, D, E and F classification are put in order as follows:

$$
\begin{gathered}
{\left[\begin{array}{l}
S_{1} \\
S_{2} \\
S_{3} \\
S_{4} \\
S_{5} \\
S_{6}
\end{array}\right]=\left[\begin{array}{llll}
a_{10} & a_{11} & a_{12} & a_{13} \\
a_{20} & a_{21} & a_{22} & a_{23} \\
a_{30} & a_{31} & a_{32} & a_{33} \\
a_{40} & a_{41} & a_{42} & a_{43} \\
a_{50} & a_{51} & a_{52} & a_{53} \\
a_{60} & a_{61} & a_{62} & a_{63}
\end{array}\right] *\left[\begin{array}{c}
1 \\
\ln v \\
\ln f \\
\ln d
\end{array}\right] \text { and }} \\
{\left[\begin{array}{l}
F_{1} \\
F_{2} \\
F_{3} \\
F_{4} \\
F_{5} \\
F_{6}
\end{array}\right]=\left[\begin{array}{llll}
b_{10} & b_{11} & b_{12} & b_{13} \\
b_{20} & b_{21} & b_{22} & b_{23} \\
b_{30} & b_{31} & b_{32} & b_{33} \\
b_{40} & b_{41} & b_{42} & b_{43} \\
b_{50} & b_{51} & b_{52} & b_{43} \\
b_{60} & b_{61} & b_{62} & b_{63}
\end{array}\right] *\left[\begin{array}{c}
1 \\
\ln v \\
\ln f \\
\ln d
\end{array}\right]}
\end{gathered}
$$

where $S_{i}$ and $F_{i}(i=1,2, \ldots, 6)$ are the logarithm values of the spindle currents $I_{s}$ and $I_{F}$ (ln represents the natural logarithm ).

\section{Fuzzy classification}

Spindle and feed current signal models at the different wear states are established The models can then be used to estimate tool wear states by the known spindle current signal, feed current signal and cutting parameters.

Measured currents $S_{0}$ and $F_{0}$ are defined as real feature values. The estimated current values $S_{i}$ and $F_{i}$ $(i=1,2, \ldots, 6)$ are defined as the cluster centers of the different tool wear classifications. The real feature values $S_{0}$ and $F_{0}$ are compared with the estimated features $S_{i}$ and $F_{i}$ by a fuzzy classification method. The membership degree of the different tool wear classifications are calculated below:

(1) if $S_{0}<S_{1}$ then

$$
\left\{\begin{array}{l}
\mu_{\mathrm{A}}(w)=1 \\
\mu_{\mathrm{B}}(w)=\mu_{\mathrm{C}}(w)=\mu_{\mathrm{D}}(w)=\mu_{\mathrm{E}}(w)=\mu_{\mathrm{F}}(w)=0
\end{array}\right.
$$

(2) if $S_{1} \leqslant S_{0}<S_{2}$ then

$$
\left\{\begin{array}{l}
\left.\left.\mu_{A}(w)=\left(S_{2}-S_{0}\right) /\right) S_{2}-S_{1}\right) \\
\mu_{B}(w)=\left(S_{0}-S_{1}\right) /\left(S_{2}-S_{1}\right) \\
\mu_{C}(w)=\mu_{D}(w)=\mu_{E}(w)=\mu_{F}(w)=0
\end{array}\right.
$$

(3) if $S_{2} \leqslant S_{0}<S_{3}$ then

$$
\left\{\begin{array}{l}
\mu_{A}(w)=\mu_{D}(w)=\mu_{E}(w)=\mu_{F}(w)=0 \\
\mu_{B}(w)=\left(S_{3}-S_{0}\right) /\left(S_{3}-S_{2}\right) \\
\mu_{C}(w)=\left(S_{0}-S_{2}\right) /\left(S_{3}-S_{2}\right)
\end{array}\right.
$$

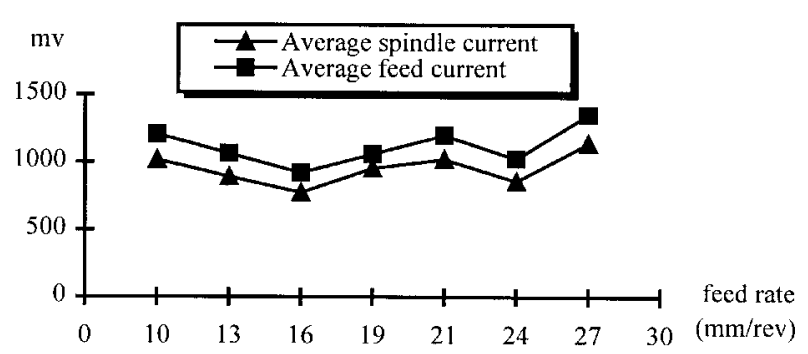

Figure 3. The effects of feed speed on current signal.

Table 2. Classifications of tool wear in boring.

\begin{tabular}{lcccccc}
\hline Classification & A & B & C & D & E & F \\
\hline Tool wear value $(\mathrm{mm})$ & $0-0.2$ & $0.1-0.3$ & $0.2-0.4$ & $0.3-0.5$ & $0.4-0.6$ & $0.5-1$ \\
\hline
\end{tabular}


(4) if $S_{3} \leqslant S_{0}<S_{4}$ then

$$
\left\{\begin{array}{l}
\mu_{A}(w)=\mu_{D}(w)=\mu_{E}(w)=\mu_{F}(w)=0 \\
\mu_{C}(w)=\left(S_{4}-S_{0}\right) /\left(S_{4}-S_{2}\right) \\
\mu_{C}(w)=\left(S_{0}-S_{3}\right) /\left(S_{4}-S_{3}\right)
\end{array}\right.
$$

(5) if $S_{4} \leqslant S_{0}<S_{5}$ then

$$
\left\{\begin{array}{l}
\mu_{A}(w)=\mu_{B}(w)=\mu_{C}(w)=\mu_{F}(w)=0 \\
\mu_{D}(w)=\left(S_{5}-S_{0}\right) /\left(S_{5}-S_{4}\right) \\
\mu_{E}(w)=\left(S_{0}-S_{4}\right) /\left(S_{5}-S_{4}\right)
\end{array}\right.
$$

(6) if $S_{5} \leqslant S_{0}<S_{6}$ then

$$
\left\{\begin{array}{l}
\mu_{A}(w)=\mu_{B}(w)=\mu_{C}(w)=\mu_{D}(w)=0 \\
\mu_{E}(w)=\left(S_{6}-S_{0}\right) /\left(S_{6}-S_{5}\right) \\
\mu_{F}(w)=\left(S_{0}-S_{5}\right) /\left(S_{6}-S_{5}\right)
\end{array}\right.
$$

(7) if $S_{6}<S_{1}$ then

$$
\left\{\begin{array}{l}
\mu_{A}(w)=\mu_{B}(w)=\mu_{C}(w)=\mu_{D}(w)=\mu_{E}(w)=0 \\
\mu_{F}(w)=1
\end{array}\right.
$$

where $\mu_{i}(w)(i=\mathrm{A}, \mathrm{B}, \ldots, \mathrm{F})$ is the membership degree of current $S_{0}$ under different tool wear classifications. The same method is fitted to the feed current.

\section{Multi-parameter fusion with fuzzy inference}

The membership degree of tool we ar states i.e. $\mu_{i}^{s}(\mathrm{w})$ and $\mu_{i}^{F}(\mathrm{w}) .(i=\mathrm{A}, \mathrm{B}, \ldots, \mathrm{F})$ with spindle current and feed current had been calculated using fuzzy classification. The above two parameters can be fused by fuzzy inference to obtain the tool wear value accurately.

\subsection{Fusion}

The relationship between the input and output variables of the fuzzy system is defined by a set of linguistic statements, which are called fuzzy rules. There are two in put variables and one output variable, which are classified into six fuzzy sets. Based on the experimental results, 26 rules, as shown in table 4 , have been developed for tool wear states fusion. These rules are classified in to six groups corresponding to six tool wear states.

Through the use of a fuzzy $\min -\max$ algorith $\mathrm{m}$, i.e. fuzzy intersection (AND) and fuzzy union (OR), the following equation can be generated to calculate the fuzzy membership values of tool wear states:

$$
\mu_{i}(w)=\bigcup_{j=1}^{K}\left\{\mu_{i}^{S}(w) \bigcap \mu_{i}^{F}(w)\right\}
$$

where $\mu_{\mathrm{i}}(w)(i=A, B, \ldots, F)$ is the fuzzy membership of tool wear states under the A, B, C, D, E and F classification, and $j=1,2, K$ represents the number of rules fired for the corresponding tool wear states.

\begin{tabular}{|c|c|c|c|c|c|c|}
\hline \multirow{2}{*}{$\begin{array}{l}\text { IF } \\
\text { rules }\end{array}$} & 1 & 2 & 3 & 4 & 5 & 6 \\
\hline & $W_{S} W_{F}$ & $W_{S} W_{F}$ & $W_{S} W_{F}$ & $W_{S} W_{F}$ & $W_{S} W_{F}$ & $W_{S} W_{F}$ \\
\hline$a$ & A A & B B & $\mathrm{C} \quad \mathrm{C}$ & $\mathrm{D} \quad \mathrm{D}$ & E E & $\mathrm{F} \quad \mathrm{F}$ \\
\hline$b$ & A B & B A & C B & $\mathrm{D} \quad \mathrm{C}$ & E D & F E \\
\hline$c$ & B A & A B & B C & C D & $\mathrm{D} E$ & E F \\
\hline$d$ & & $\mathrm{~B} \quad \mathrm{C}$ & C D & $\mathrm{D} \quad \mathrm{E}$ & E F & \\
\hline$e$ & & C B & $\mathrm{D} \quad \mathrm{C}$ & E D & F E & \\
\hline Wear states & A & B & C & $\mathrm{D}$ & $\mathrm{E}$ & $\mathrm{F}$ \\
\hline
\end{tabular}

Table 3. Constants of fuzzy membership functions for tool we ar condition.

\begin{tabular}{lrrrr}
\hline & \multicolumn{3}{c}{ Constants of fuzzy membership function } \\
\cline { 2 - 5 } $\begin{array}{l}\text { Tool wear } \\
\text { classification }\end{array}$ & $a$ & $b$ & $k$ & $l$ \\
\hline A & 0 & 1 & 0 & 0.15 \\
& 20 & 4 & 0.15 & 0.20 \\
B & 20 & -3 & 0.15 & 0.20 \\
& 0 & 1 & 0.20 & 0.25 \\
& 20 & 6 & 0.25 & 0.30 \\
C & 20 & -5 & 0.25 & 0.30 \\
& 0 & 1 & 0.30 & 0.35 \\
& 20 & 8 & 0.35 & 0.40 \\
D & 20 & -7 & 0.35 & 0.40 \\
& 0 & 1 & 0.40 & 0.45 \\
& 20 & 10 & 0.45 & 0.50 \\
E & 20 & -9 & 0.45 & 0.50 \\
& 0 & 1 & 0.50 & 0.55 \\
& 20 & 12 & 0.55 & 0.6 \\
F & 20 & -11 & 0.55 & 0.6 \\
& 0 & 1 & 0.6 & 0.7 \\
\hline
\end{tabular}

Table 4. Fuzzy rules for tool wear states fusion.

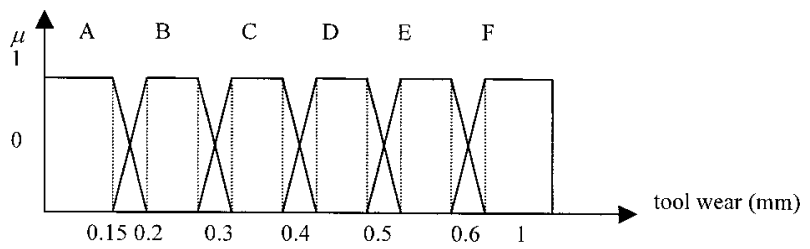

Figure 4. Fuzzy membership of tool wears state. 


\subsection{Obtaining (fuzzy) tool wear value}

The key to the fusion of tool wear states is the selection of appropriate shapes of fuzzy membership for process variables based on experimental results. Figure 4 shows the membership function of tool wear states. The reason for choosing trapezoidal shape for tool wear states is that is difficult to quantity an exact wear value. Using a wider range avoids defining an exact wear value for a certain level of linguistic variable of tool wear. This will also allow an easy knowledge acquisition when developing a set of fuzzy rules for fuzzy inference. Based on the classification of tool wear states, the trapezoid function is defined as follows:

$$
\mu(w)=a w+b \quad k<w<l
$$

where $\mu(w)$ is the fuzzy membership value for tool wear states, and $a, b, k$ and $l$ are constan ts for different fuzzy sets as shown in table 4.

\subsection{Defuzzification of tool wears}

The outputs of the inference process are still fuzzy values and they need to be defuzzified. Basically, defuzzification is a mapping from a space of fuzzy values into that of the non-fuzzy universe. At present, there are several strategies which can be used to perform the defuzzification process. The most commonly used strategy is the centred defuzzy method (Lee 1990), which produces the center of area of the possibility distribution of inference output. Therefore, the defuzzified tool wear states can be obtained by using the formula:

$$
\text { wear }=\frac{\int_{w} \mu(w) w \mathrm{~d} w}{\int_{w} \mu(w) \mathrm{d} w}
$$

where wear represents the numerical value of tool wear states and $\mu(w)$ is the fuzzy membership degree fused by fuzzy inference.

\section{Results and discussion}

A total of 77 tool wear cutting tests are collected under various cutting conditions. 50 samples are randomly picked as learning samples; 27 samples are used as the test samples in the classification phase. According to the classification of the tool wear states, 50 learning samples are divided into 6 groups. The $a_{i j}$ and $b_{i j}$ values of (2) are calculated by the least square method; the results are as follows:

$\left(\begin{array}{llll}5.2623 & 0.3206 & 0.0835 & 0.0928 \\ 5.0250 & 0.3477 & 0.1512 & 0.0366 \\ 5.3582 & 0.2532 & 0.2228 & 0.1801 \\ 5.5319 & 0.1993 & 0.2723 & 0.2383 \\ 6.3176 & 0.0138 & 0.2733 & 0.3425 \\ 6.4744 & -0.0249 & 0.3205 & 0.4285\end{array}\right)$ and
$\left(\begin{array}{cccc}5.6904 & 0.2480 & 0.0916 & 0.0688 \\ 5.4995 & 0.2770 & 0.1385 & 0.0163 \\ 5.7568 & 0.2060 & 0.1945 & 0.1295 \\ 5.9368 & 0.1553 & 0.2315 & 0.1900 \\ 7.4501 & -0.1591 & 0.1151 & 0.4199 \\ 6.7410 & -0.0318 & 0.2736 & 0.3820\end{array}\right)$

The correlation coefficient that correspond to the weight value of each group are: $0.9026,0.8240,0.7938$, $0.7923,0.9169,0.9746,0.9062,0.8089,0.7805,0.7727$, 0.9179 and 0.9680 . It is obvious that the correlation coefficients are very close to unity. It is indicated that the relationship between the current signals and the cutting parameters is well represented by the proposed models.

\begin{tabular}{|c|c|c|c|c|c|c|}
\hline & $\begin{array}{l}\text { Spindle } \\
\text { speed } \\
(\mathrm{m} / \mathrm{min})(\end{array}$ & $\begin{array}{c}\text { Feed } \\
\text { speed } \\
\mathrm{mm} / \mathrm{min})\end{array}$ & $\begin{array}{l}\text { The } \\
\text { depth of } \\
\text { cut } \\
(\mathrm{mm})\end{array}$ & $\begin{array}{l}\text { Wear } \\
\text { value } \\
(\mathrm{mm})\end{array}$ & $\begin{array}{l}\text { Spindle } \\
\text { current } \\
(\mathrm{mv})\end{array}$ & $\begin{array}{c}\text { Feed } \\
\text { current } \\
(\mathrm{mv})\end{array}$ \\
\hline 1 & 15 & 10 & 0.35 & 0.11 & 479.0 & 629.0 \\
\hline 2 & 15 & 10 & 0.35 & 0.24 & 536.0 & 695.0 \\
\hline 3 & 15 & 10 & 0.35 & 0.32 & 608.5 & 792.5 \\
\hline 4 & 15 & 10 & 0.35 & 0.41 & 685.0 & 873.0 \\
\hline 5 & 15 & 10 & 0.35 & 0.56 & 836.0 & 1027.0 \\
\hline 6 & 15 & 20 & 0.55 & 0.17 & 623.5 & 802.5 \\
\hline 7 & 15 & 20 & 0.55 & 0.34 & 682.0 & 865.0 \\
\hline 8 & 15 & 20 & 0.55 & 0.37 & 721.5 & 895.5 \\
\hline 9 & 15 & 20 & 0.55 & 0.41 & 845.0 & 1008.0 \\
\hline 10 & 15 & 20 & 0.55 & 0.49 & 995.0 & 1177.0 \\
\hline 11 & 15 & 20 & 0.55 & 0.68 & 1428.0 & 1597.0 \\
\hline 12 & 15 & 30 & 0.90 & 0.26 & 757.0 & 947.0 \\
\hline 13 & 15 & 30 & 0.90 & 0.36 & 880.5 & 1032.5 \\
\hline 14 & 15 & 30 & 0.90 & 0.41 & 1190.0 & 1343.0 \\
\hline 15 & 15 & 30 & 0.90 & 0.53 & 1461.5 & 1620.5 \\
\hline 16 & 25 & 10 & 0.50 & 0.13 & 576.0 & 762.0 \\
\hline 17 & 25 & 10 & 0.50 & 0.27 & 654.0 & 828.0 \\
\hline 18 & 25 & 10 & 0.50 & 0.45 & 923.5 & 1117.5 \\
\hline 19 & 25 & 10 & 0.50 & 0.74 & 1022.5 & 1210.5 \\
\hline 20 & 25 & 20 & 1.15 & 0.27 & 940.5 & 1096.5 \\
\hline 21 & 25 & 20 & 1.15 & 0.61 & 1572.5 & 1753.5 \\
\hline 22 & 25 & 30 & 0.45 & 0.27 & 702.5 & 868.5 \\
\hline 23 & 25 & 30 & 0.45 & 0.31 & 978.5 & 1177.5 \\
\hline 24 & 25 & 30 & 0.45 & 0.46 & 1176.0 & 1357.0 \\
\hline 25 & 35 & 10 & 0.90 & 0.51 & 1265.0 & 1434.0 \\
\hline 26 & 35 & 20 & 0.50 & 0.64 & 1121.0 & 1303.0 \\
\hline 27 & 35 & 30 & 0.80 & 0.44 & 1161.5 & 1360.5 \\
\hline
\end{tabular}

Table 5. Test sample. 
Table 6. Membership degrees of tool wear states.

Membership degree

\begin{tabular}{|c|c|c|c|c|c|c|c|}
\hline Test & $\mu_{A}$ & $\mu_{B}$ & $\mu_{C}$ & $\mu_{D}$ & $\mu_{E}$ & $\mu_{F}$ & $\begin{array}{l}\text { Estimated } \\
\text { wear value }(\mathrm{mm})\end{array}$ \\
\hline 1 & 1 & & 0.0819 & & & & 0.100 \\
\hline 2 & 0.1513 & 0.8487 & 0.4745 & 0.5255 & & & 0.210 \\
\hline 3 & & & & 0.5120 & 0.5120 & & 0.378 \\
\hline 4 & & & & & & 1 & 0.475 \\
\hline 5 & & & & & & & 0.600 \\
\hline 6 & & 0.8135 & 0.1865 & & & & 0.239 \\
\hline 7 & & 0.4062 & 0.5930 & & & & 0.286 \\
\hline 8 & & 0.1463 & 0.8537 & & & & 0.314 \\
\hline 9 & & 0.1926 & 0.8074 & & & & 0.310 \\
\hline 10 & & & & 0.3191 & 0.689 & & 0.497 \\
\hline 11 & & & & & & 1 & 0.600 \\
\hline 12 & & 0.4787 & 0.5213 & & & & 0.273 \\
\hline 13 & & 0.1043 & 0.8957 & & & & 0.318 \\
\hline 14 & & & & 0.6372 & 0.3628 & & 0.458 \\
\hline 15 & & & & & 0.8247 & 0.1573 & 0.528 \\
\hline 16 & 1 & & & & & & 0.1 \\
\hline 17 & & 0.7516 & 0.2484 & & & & 0.245 \\
\hline 18 & & & & & 0.0899 & 0.9101 & 0.592 \\
\hline 19 & & & & & & 1 & 0.600 \\
\hline 20 & & 0.1075 & 0.8925 & & & & 0.317 \\
\hline 21 & & & & & 0.3094 & 0.6906 & 0.570 \\
\hline 22 & 0.6114 & 0.3886 & & & & & 0.333 \\
\hline 23 & & & & 0.7418 & 0.7148 & & 0.475 \\
\hline 24 & & & & & 0.5976 & 0.4024 & 0.542 \\
\hline 25 & & & & & & 1 & 0.600 \\
\hline 26 & & & & & 0.2774 & 0.7226 & 0.573 \\
\hline 27 & & & & 0.5760 & 0.5760 & & 0.475 \\
\hline
\end{tabular}

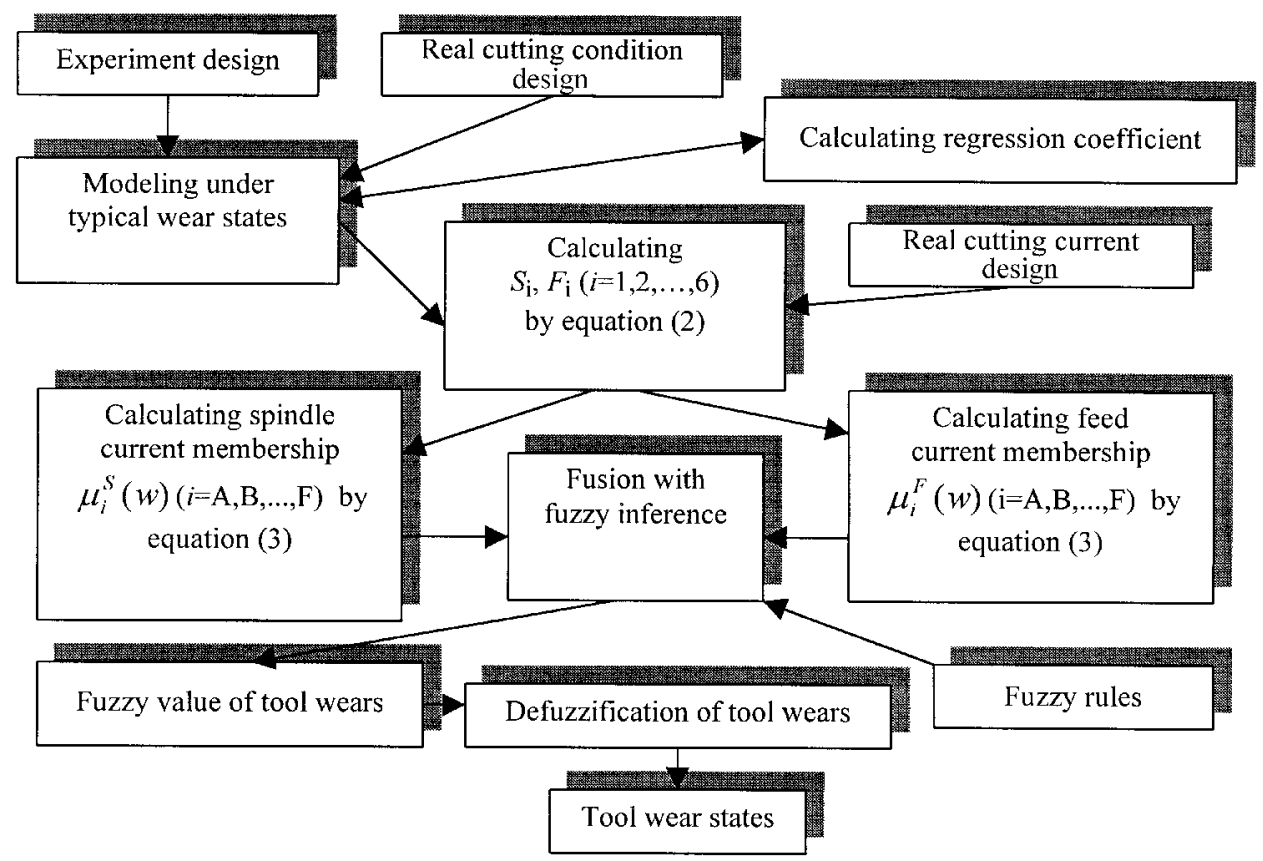

Figure 5. Flowchart of the tool wear states recognition method. 
tool wear value $(\mathrm{mm})$

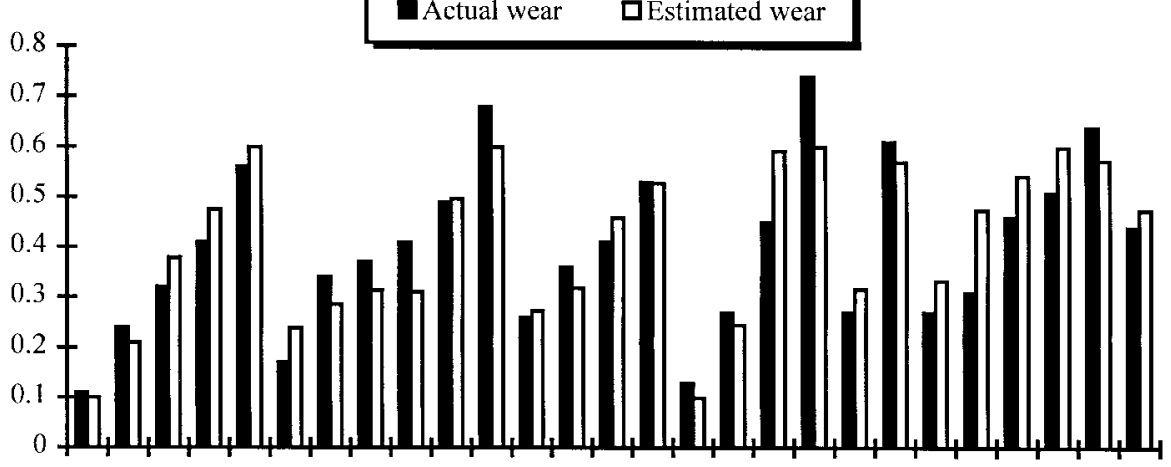

Figure 6. The comparison of actual tool wear with estimated tool wear.

Twenty-seven additional tests are conducted to examine the feasibility of using the above models to estimate tool wear states. The cutting parameters and measured current signals for these additional tests are shown in table 5 .

The above method is used to estimate the tool wear value. First, the logarithms of the present cutting parameters $v, f$ and $d$, as well as 1 , are put into (2); the estimated values of the spindle and feed currents, namely $S_{i}$ and $F_{i}(i=1,2, \ldots, 6)$ are output. Secondly, the spindle and feed current values detected are 'logged', then compared with $S_{i}$ and $F_{i}$. The membership degrees of present tool wear states under the different tool wear classification $\mathrm{s}$ are calculated based on (3). Finally, the membership degrees of the tool states are fused by fuzzy inference; the accurate tool wear value is detected using the centered defuzzy method. The results of the membership degrees of the tool states fused and the estimated tool wear values are in table 6. The above processing can briefly be expressed in figure 5. In order to make clear the reliability of the above method, a comparison of the actual tool wear values with those estimated is shown in figure 6 . The results show that above method has more accurately estimated the tool wear states.

\section{Conclusion}

A new method of tool wear states identification is presented. It can effectively identify a tool wear value with the detected current signal. The following conclusions are drawn.

The effect of tool wear and cutting parameters on the spindle and feed current signals is analyzed. The models of the relationship between the current signals and the cutting parameters under diffe rent tool wear states have been established for different cutting conditions using experimental design and regression analysis.
The fuzzy classification method has been successfully used to calculate the membership of different tool wear states by the detected current value.

Fuzzy inference is used to fuse the membership degree of tool wear states with the spindle current and feed current; tool wear value is finally obtained.

Experimental results show that the method can be effectively employed in practice. The algorithm is easy and reliable, and the detecting system can be applied at almost no extra cost. In addition, not only can the method fit with the current signal, but it can also fit with the force signal, etc. Therefore, the method could be developed to other tool condition monitoring systems.

\section{References}

Byrne, G., Dornfeld, D., Inasaki, I., Ketteler, G., Konig, W. and Teti, R., 1995, Tool condition monitoring ( TCM)-the status of research and industrial application. Annals of the CIRP, 44, $541-567$.

Daneshmand, L. K. and PAK, H. A., 1983, Performance monitoring of a computer numerically controlled (CNC) lathe using pattern recognition techniques. Third International Conference on Robot Vision and Sensor Controls, Cambridge, MA.

Lee, C. C., 1990, Fuzzy logic in control systems fuzzy logic controller-Part II. IEEE Transactions on Systems, Man and Cybernetics, 20, $419-435$.

Li, D., and Mathew, J., 1990, Tool wear and failure monitoring techniques for turning-a review. International Journal of Machine Tools Manufacturing, 30, 579 - 598.

Mannan, M. A., Broms, S. and Lindustrom, B., 1989, Monitoring and adaptive control of cutting process by means of motor power and current measurements. Annals of the CIRP, 38, $347-350$.

Mannan, M. A. and Nilsson, T., 1997, The behavior of static torque and thrust due to tool wear in boring. Technical papers of the North American Manufacturing Research Institution of $S M E$, pp. $75-80$.

Shaw, M.C., 1984. Metal Cutting Principle (Oxford University Press). 